\title{
The Use of Antidepressants During Pregnancy
}

\section{ISSN: 2640-9666}

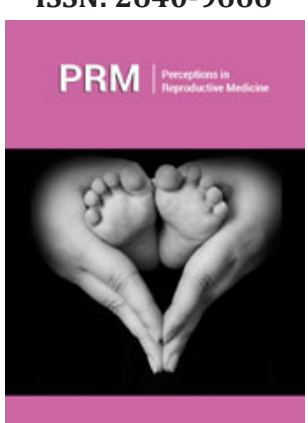

*Corresponding author: Hani Raoul Khouzam, Staff Psychiatrist, Mental Health and Psychiatry Services, Clinical Professor of Psychiatry and Medicine, California, USA

Submission: 愠 May 22, 2021

Published: 海June 18, 2021

Volume 4 - Issue 4

How to cite this article: Hani Raoul Khouzam. The Use of Antidepressants During Pregnancy. Perceptions Reprod Med. 4(3). PRM. 000593. 2021.

DOI: 10.31031/PRM.2021.04.000593

Copyright@ Hani Raoul Khouzam, This article is distributed under the terms of the Creative Commons Attribution 4.0 International License, which permits unrestricted use and redistribution provided that the original author and source are credited.

\author{
Hani Raoul Khouzam ${ }^{1,2 *}$ \\ ${ }^{1}$ Staff Psychiatrist, Mental Health and Psychiatry Services, USA \\ ${ }^{2}$ Clinical Professor of Psychiatry and Medicine, USA
}

\begin{abstract}
Depression is common during pregnancy. This review summarizes the risks associated with depression during pregnancy for the mothers and developing fetus, and the treatment of depression with antidepressants during pregnancy.
\end{abstract}

Keywords: Pregnancy; Depression; Antidepressants; Risks; Treatment

Abbreviations: SSRIs: Selective Serotonin Reuptake Inhibitors; SNRIs: Serotonin and Norepinephrine Reuptake Inhibitors; TCAs: Tricyclic Antidepressants

\section{Introduction}

Depression is common during the childbearing years for women aged 15-44 years and it is estimated to affect $14.5 \%$ of pregnant women [1]. Untreated maternal depression could have a detrimental effect on the pregnant women and their developing fetus. However there seem to be a decreased accuracy in diagnosing and treating depression during pregnancy [2]. There is also a reluctance by pregnant women and clinicians to use antidepressants due to fears of their potential harmful effects on the pregnancy and the growing fetus [3]. The accurate diagnosis of depression and its treatment with antidepressants while weighing on their potential risks and benefits would lead to an improved clinical outcome for pregnant mother, the growing fetus, and the newborn [4].

\section{Diagnosing Depression during Pregnancy}

Depression can often be overlooked in pregnant women due to the similarity of its symptoms with the physiological disturbances associated with pregnancy such as sleep and appetite changes, diminished energy, and the marked decreased libido. Therefore, clinicians should evaluate the psychological rather than the physiological manifestation of depression such as a lack of interest in the pregnancy, ruminations of guilt, anhedonia, the presence of passive death wish, or the emergence of suicidal ideation [5]. To accurately identify depression, clinicians caring for pregnant women can use the various screening tools that are specifically designed to recognize depression during pregnancy. These may include the Primary Care Evaluation of Mental Disorders Patient Health Questionnaire (PRIME-MD,PHQ) [6], the Edinburgh Postnatal Depression Scale (EPDS) [7], the Beck Depression Inventory Scale (BDI-II) [8] and the Center for Epidemiologic Studies Depression Scale, Revised (CESDR) [9]. The diagnostic and statistical manual of mental disorders criteria can also be used to confirm the diagnosis of depression regardless of pregnancy status [10].

\section{Risks for Depression in Pregnancy}

The development of depression during pregnancy could be triggered by several psychosocial stressors such as exposure to trauma and violence, relationship conflicts, and inadequate social support systems. Poverty, food, housing and financial insecurities may 
further exacerbate these stressors. Single motherhood, unplanned or unexpected pregnancy, and having several infants and toddlers could also precipitate depression [11]. Early age pregnancy especially during adolescence also increases of the incidence depression [12]. A personal or family history of depressive illness could also predispose to depression during pregnancy [13]. The discontinuation of antidepressant medications would frequently lead to the recurrence or the worsening of preexisting depression [14]. Quality care for pregnant women would need to implement clinical guidelines that could identify and initiate psychosocial interventions to address and reverse these depression predisposing risk factors.

\section{Risks Associated with Untreated Maternal Depression}

Maternal depression does not only affect the wellbeing of the pregnant mother but could have detrimental effects on the growing fetus and could lead to a worsening of pregnancy outcomes. Untreated maternal depression could have catastrophic effects such as suicide and, or infanticide $[15,16]$. Untreated depression during pregnancy has been associated with increase alcohol, tobacco and illicit substance use which would lead to negative consequences in the mother and the growing fetus [17]. Malnutrition, poor adherence with prenatal care and failure to recognize or report the early signs of labor are also some of the sequelae of maternal depression resulting in poor obstetric, fetal, and neonatal outcomes [18]. Increased levels of discomfort and multiple somatic complaints such as headache, nausea, stomach pain, shortness of breath, gastrointestinal problems, palpitations, dizziness, and sexual dysfunction, could also be manifestations of unrecognized depression often leading to unwarranted diagnostic procedures and medical interventions [18]. Global functional impairment, pre-eclampsia, increased risk of postnatal depression and ultimately poor pregnancy outcomes have all been associated with untreated maternal depression [19].

\section{Effects of Maternal Depression on the Fetus and the Newborn}

Maternal depression has been associated with premature birth, low birth weight, and fetal growth restriction, and postnatal cognitive and emotional complications [4]. Newborns of depressed mothers have been reported to have increased irritability, fewer facial expressions and could be at risk for developmental delay [4]. Neonates whose mothers are depressed are more likely to have lower Apgar scores and a smaller head circumference [11]. Infants of depressed mothers may experience dysregulation of their hypothalamic-pituitary-adrenal axis and higher admission rates to neonatal intensive care units [20]. As they develop, these neonates may have more difficulty in engaging in social interactions, show less positive and more negative affect, and have worse developmental and emotional outcomes [21].

\section{Antidepressants Treatment During Pregnancy}

Antidepressant medications are generally considered safe in the treatment of depression during pregnancy. They should be seriously considered if the clinical assessment confirms the presence of moderate to severe symptoms of depression, that are affecting daily functioning and impairing the ability to care for self and adherence with obstetric prenatal care. The reinitiating of the same antidepressant that was discontinued due to the pregnancy should be considered as a first option based on prior benefits that were achieved by that medication. Available evidence suggests that the Selective Serotonin Reuptake Inhibitors (SSRIs) are generally considered an option during pregnancy, including fluoxetine sertraline and citalopram [22,23]. Most studies show that SSRIs are not associated with birth defects [24]. Some concerns have been raised regarding paroxetine being associated with a possible increased risk of a fetal heart defect and pulmonary hypertension thus discouraging its use during pregnancy [25].

The Serotonin and Norepinephrine Reuptake Inhibitors (SNRIs) such as venlafaxine and duloxetine could also be considered as another option during pregnancy the safety of duloxetine however has not been fully researched [26]. Mirtazapine may be a viable option for use in pregnancy because unlike the SSRIs and SNRIs it is not associated with nausea, and it may be beneficial in some patients with hyperemesis gravidarum [27]. It can cause weight gain which could predispose to possible obstetrical complications such as gestational diabetes, also its sedating properties may be cumbersome in pregnancy. It is still unclear if mirtazapine increases the risk of fetal malformations, and spontaneous abortion [27]. Bupropion although not generally considered a first line treatment it may be a reasonable treatment option during pregnancy, particularly as an adjunctive agent that helps with smoking cessation [28].

Tricyclic Antidepressants (TCAs) such as nortriptyline and desipramine were widely utilized in pregnancy prior to the introduction of SSRIs and SNRIs. Some studies that have not been confirmed suggested that limb anomalies could occur with TCAs in pregnancy. Some infants exposed to TCAs exhibited acute effects that were similar to neonatal abstinence syndrome which could be manifested by tachypnea, tachycardia, cyanosis, irritability, hypertonia, and clonus. Current clinical guidelines do not recommend TCAs and as first line or second line treatment but could be used as an option during pregnancy for those who have not responded to the SSRIs ,SNRIs or other antidepressants.

\section{Conclusion}

Untreated maternal depression during pregnancy could have detrimental effects on the mother and the developing fetus and has been associated with premature birth, low birth weight, fetal growth restriction, and postnatal cognitive and emotional complications. Treating depression during pregnancy with antidepressants is considered generally safe and outweigh the risks associated with their potential side effects. High quality pregnancy care should include assessment tools to accurately recognize, diagnose and treat depression. Antidepressant medications are viable treatment options for moderate and severe depression for pregnant mothers who have not responded to implemented social and psychotherapeutic interventions. Fully informed treatment 
decision-making requires balancing the risks and benefits of proposed interventions against those of untreated depression.

\section{Acknowledgments}

Sincere gratitude and thankfulness to my wife Lynn and children, Andrew, Adam, Andrea and her husband Nic, and their daughter Abigail, my sisters Hoda and Héla, and my brother Hadi for their support and encouragement.

\section{References}

1. Marcus SM, Flynn HA, Blow FC, Barry KL (2003) Depressive symptoms among pregnant women screened in obstetrics settings. J Womens Health (Larchmt) 12(4): 373-380.

2. Bennett HA, Einarson A, Taddio A, Koren G, Einarson TR (2004) Prevalence of depression during pregnancy: Systematic review. Obstet Gynecol 103(4): 698-709.

3. Stewart DE (2011) Clinical practice. Depression during pregnancy. N Engl J Med 365(17): 1605-1611.

4. Grote NK, Bridge JA, Gavin AR, Melville JL, Iyengar S, et al. (2010) A meta-analysis of depression during pregnancy and the risk of preterm birth, low birth weight, and intrauterine growth restriction. Arch Gen Psychiatry 67(10): 1012-1024.

5. Romm A (2014) Diagnosing and treating depression during pregnancy. Alternative and Complementary Therapies 20(2): 76-79.

6. Spitzer RL, Kroenke K, Willaims JB (1999) Validation and utility of a self-report version of PRIME-MD: The PHQ Primary Care Study. JAMA 282(2): 1737-1744.

7. Cox JL, Holden JM, Sagovsky R (1987) Detection of postnatal depression. Development of the 10-item edinburgh postnatal depression scale. Br J Psychiatry 150: 782-786.

8. Feinman JA, Cardillo D, Palmer J, Mitchel M (2000) Development of a model for the detection and treatment of depression in primary care. Psychiatr Q 71(1): 59-78.

9. Radloff L (1977) The CES-D scale: A self-report depression scale for research in the general population. Applied Psychological Measurement 1(3): 385-401.

10. American Psychiatry Association (2013) Diagnostic and statistical manual of mental disorders ( $5^{\text {th }}$ edn), American Psychiatric Association Publishing, Inc; Washington, DC, USA.

11. Cohen LS, Nonacs RM (2005) Mood and anxiety disorders during pregnancy and postpartum. American Psychiatric Association Publishing, Inc, Washington, DC, USA.

12. Dietz PM, Williams SB, Callaghan WM, Bachman DJ, Whitlock EP, et al. (2007) Clinically identified maternal depression before, during, and after pregnancies ending in live births. Am J Psychiatry 164(10): 15151520 .
13. Gotlib IH, Whiffen VE, Mount JH, Milne K, Cordy NI, et al. (1989) Prevalence rates and demographic characteristics associated with depression in pregnancy and the postpartum. J Consult Clin Psychol 57(2): 269-274.

14. Cohen LS, Altshuler LL, Harlow BL, Nonacs R, Newport J, et al. (2006) Relapse of major depression during pregnancy in women who maintain or discontinue antidepressant treatment. JAMA 295(5): 499-507.

15. Rhodes AM, Segre LS (2013) Perinatal depression: A review of US legislation and law. Arch Womens Ment Health 16(4): 259-270.

16. Friedman SH, Horwitz SM, Resnick PJ (2005) Child murder by mothers: A critical analysis of the current state of knowledge and a research agenda. Am J Psychiatry 162(9): 1578-1587.

17. Forray A, Foster D (2015) Substance use in the perinatal period. Curr Psychiatry Rep 17(11): 91.

18. Haas JS, Afflick FE, Stewart AL, Jackson RA, Dean ML, et al. (2005) Prepregnancy health status and the risk of preterm delivery. Arch Pediatr Adolesc Med 159(1): 58-63.

19. Field T, Diego M, Hernandez-Reif M (2006) Prenatal depression effects on the fetus and newborn: A review. Infant Behav Dev 29(3): 445-455.

20. Bonari L, Pinto N, Ahn E, Einarson A, Steiner M, et al. (2004) Perinatal risks of untreated depression during pregnancy. Can J Psychiatry 49(11): 726-735.

21. Kulin NA, Pastuszak A, Sage SR, Boschetto BS, Glenda S, et al. (1998) Pregnancy outcome following maternal use of the new selective serotonin reuptake inhibitors: A prospective controlled multicenter study. JAMA 279: 609-610.

22. Addis A, Koren G (2000) Safety of fluoxetine during the first trimester of pregnancy: A meta-analytical review of epidemiological studies. Psychol Med 30(1): 89-94.

23. Ornoy A, Koren G (2017) Selective serotonin reuptake inhibitors during pregnancy: Do we have now more definite answers related to prenatal exposure? Birth Defects Res 109(12): 898-908.

24. Williams M, Wooltorton E (2005) Paroxetine (Paxil) and congenital malformations. CMAJ 173(11): 1320-1321.

25. Bellantuono C, Vargas M, Mandarelli G (2005) The safety of serotoninnoradrenaline reuptake inhibitors (SNRIs) in pregnancy and breastfeeding: a comprehensive review. Hum Psychopharmacol 30(3):143-151.

26. Djulus J, Koren G, Einarson TR, Wilton L, Shakir S, et al. (2006) Exposure to mirtazapine during pregnancy: a prospective, comparative study of birth outcomes. J Clin Psychiatry 67(8): 1280-1284.

27. Hendrick V, Suri R, Gitlin MJ, Portillo OE (2017) Bupropion use during pregnancy: A systematic review. Prim care companion. CNS Disord 19(5): 17

28. Gentile S (2014) Tricyclic antidepressants in pregnancy and puerperium. Expert Opin Drug Saf 13(2): 207-225. 